\title{
Failure of medical therapy for pulmonary "thromboembolic" disease: Beware the unsuspected primary sarcoma of the pulmonary artery
}

\author{
Cliff K. Choong, FRACS, Jennifer S. Lawton, MD, Marc R. Moon, MD, and Ralph J. Damiano, Jr, MD, St Louis, Mo
}

$\mathrm{P}$ rimary sarcoma of the pulmonary artery (PA) is rare. The diagnosis is difficult and often delayed. Two patients with primary sarcoma of the PA that mimicked pulmonary thromboembolic diseases who presented with complications requiring surgical intervention are described. The diagnostic and management issues regarding these often unsuspected but aggressive tumors are discussed.

\section{Clinical Summary}

PATIENT 1. A 54-year-old woman was admitted for pulmonary thromboendarterectomy workup. She had a past medical history of pulmonary embolism (PE) that occurred 3 months before admission. This was complicated by an area of right lower lobe infarction seen on chest computed tomography (CT) scanning. A deep vein thrombosis of her right femoral vein was diagnosed on lower extremity Doppler investigation as the cause of her PE. Her treatment consisted of anticoagulation and placement of an inferior vena cava filter. The results of all screening tests for a hypercoagulable state were negative. She had progressive shortness of breath over the following 3-month period and was in New York Heart Association class IV at the time of admission. A chest CT scan revealed a massive pulmonary embolus in the right PA (Figure 1, A). A pulmonary arteriogram demonstrated total occlusion of the right PA approximately $4 \mathrm{~cm}$ from its origin, whereas the left PA and its branch vessels were normal (Figure 1, B). Echocardiography revealed severe tricuspid regurgitation and a PA systolic pressure of $65 \mathrm{~mm} \mathrm{Hg}$. In view of her symptoms and occluded right PA, she underwent a right pulmonary endarterectomy to restore antegrade right pulmonary blood flow. Intraoperatively, extensive clots within the right PA were found and removed. The right PA had a very thickened intima consisting of tan-yellow membranous tissue and nodules that had markedly narrowed the PA lumen. A right PA endarterectomy was performed during hypothermic circulatory arrest. Her postoperative recovery was uneventful, and she was discharged 9 days later. Histopathology revealed a

From the Department of Surgery, Division of Cardiothoracic Surgery, Washington University School of Medicine, St Louis, Mo.

Received for publication March 15, 2004; accepted for publication March 30, 2004.

Address for reprints: Jennifer S. Lawton, MD, Department of Surgery, Division of Cardiothoracic Surgery, Washington University School of Medicine, Queeny Tower, Suite 3108, One Barnes-Jewish Hospital Plaza, St Louis, MO 63110 (E-mail: lawtonj@msnotes.wustl.edu).

J Thorac Cardiovasc Surg 2004;128:763-5

$0022-5223 / \$ 30.00$

Copyright (C) 2004 by The American Association for Thoracic Surgery

doi:10.1016/j.jtcvs.2004.03.049 poorly differentiated primary sarcoma arising from the wall of the PA. Immunohistochemical staining revealed that the tumor cells were strongly positive for $\alpha$-smooth muscle actin and vimentin while negative for creatine kinase and S-100. CD31 stains highlighted the presence of capillaries within the tumor. These results were consistent with a primary leiomyosarcoma of the PA. She was treated with postoperative adjuvant radiation and a chemotherapy regimen consisting of doxorubicin, ifosfamide, mesna, and dacarbazine. Mediastinal lymph node and bony metastatic disease were detected at 5-month followup, and she died 7 months later.

PATIENT 2. A 49-year-old previously healthy man presented to the emergency department with a 5-week history of progressive shortness of breath, dyspnea on exertion, and syncope while playing tennis. He had a positive ventilation-perfusion pulmonary scan result for $\mathrm{PE}$, and a large saddle embolus at the bifurcation of the main PA was seen on chest CT scanning (Figure 2). An echocardiogram revealed a PA systolic pressure of $82 \mathrm{~mm} \mathrm{Hg}$, moderate tricuspid regurgitation, a markedly dilated right ventricle, and a markedly decreased right ventricular function. There was no evidence of deep vein thrombosis on lower limb venous Doppler examination. Laboratory tests did not detect any evidence for a hypercoagulable state. He was hemodynamically stable and was treated medically with anticoagulation and subsequently discharged home. Two weeks later, he was readmitted with increasing shortness of breath and right-sided chest pain. A chest CT scan revealed 2 new areas of right-sided acute pulmonary infarcts despite a therapeutic international normalized ratio of 4.8. Because of the failure of medical therapy and severe symptoms, he underwent emergency pulmonary embolectomy with cardiopulmonary bypass. Thick, white fibrinous material was found adherent to both PAs and was removed. An inferior vena cava filter was also placed during the same hospital admission. He made a smooth postoperative recovery and was discharged home on postoperative day 6. Histopathology and immunohistochemical stains of the extracted specimen revealed a spindle cell sarcoma of the PA. The patient died 1 month later, and postmortem examination revealed tumor filling both branch PAs.

\section{Discussion}

The clinical features of primary sarcoma of the PA can be indistinguishable from those of pulmonary thromboembolic disease, as seen in both described cases. Diagnosis is seldom made preoperatively and is often unsuspected before surgical intervention for acute or chronic pulmonary obstruction. ${ }^{1}$ An associated past history of deep vein thrombosis, as seen in patient 1 , would further mislead the diagnosis to that of chronic pulmonary thromboembolic disease as the cause of the pulmonary vascular obstruction. Atypical cases of PE, such as complete unilateral obstruction of a main PA (patient 1) or recurrent pulmonary emboli despite ade- 

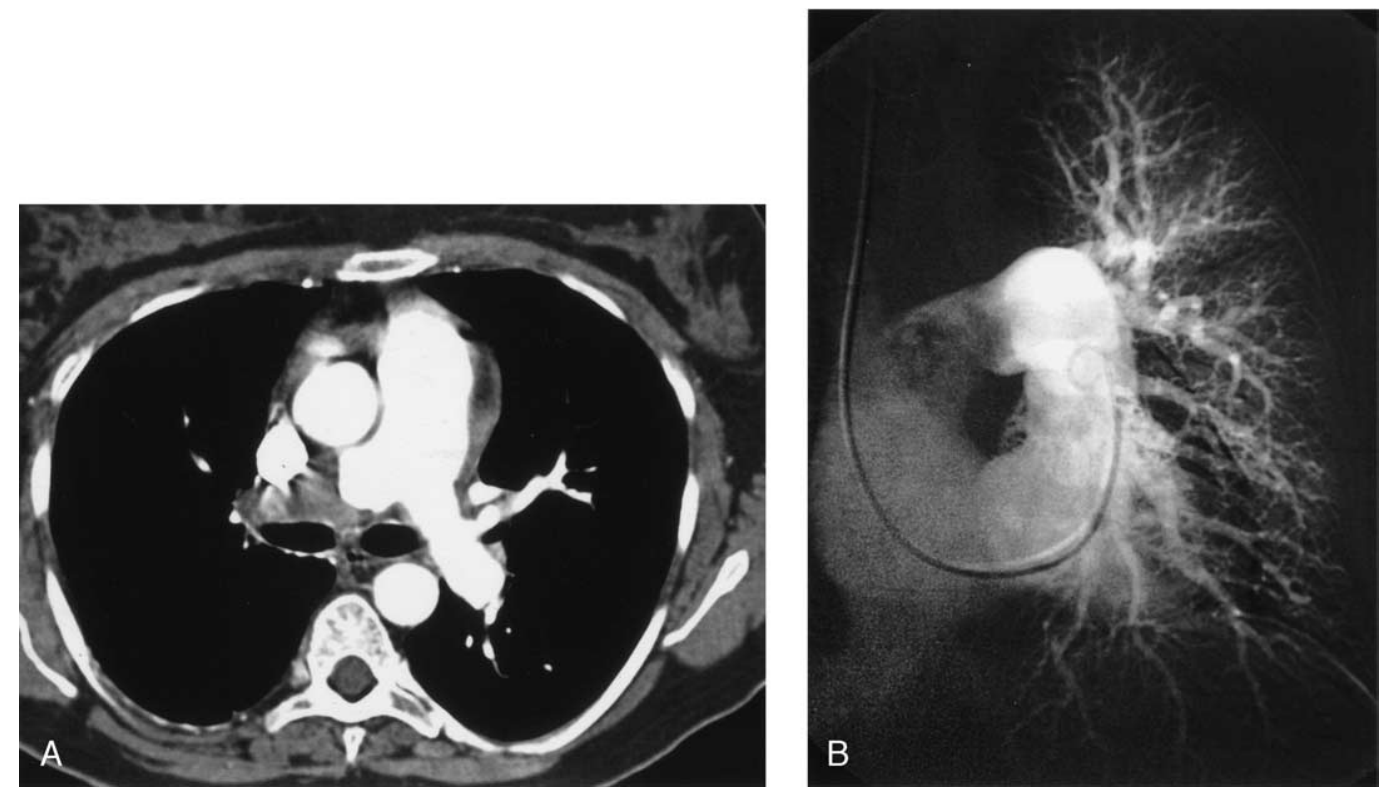

Figure 1. A, CT scan demonstrating complete occlusion of the right pulmonary artery. B, Pulmonary arteriogram showing total occlusion of the right pulmonary artery, whereas the left pulmonary artery and its branch vessels were normal.

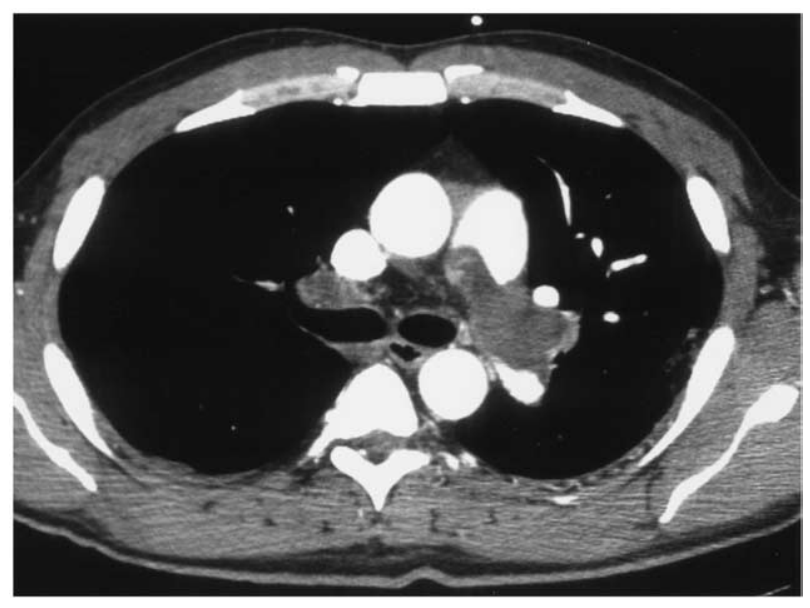

Figure 2. CT scan showing a massive saddle embolus at the bifurcation of the main pulmonary artery.

quate anticoagulation without evidence of deep vein thrombosis (patient 2), should raise a high index of suspicion for PA sarcoma. ${ }^{2}$ The radiologic diagnosis of primary PA sarcoma can still be difficult despite technological advances because it is often not possible to distinguish it from acute or chronic PE. Preoperative pulmonary angiography can show intraluminal narrowing of the PAs but is unable to distinguish between tumor and thromboembolic diseases unless atypical features such as pedunculated or lobulated lesions are seen. ${ }^{2} \mathrm{CT}$ scans in both the described patients did not demonstrate any evidence suggestive of PA malignancy. This is not unusual because CT scans cannot differentiate between thrombus and intraluminal tumor unless there is evi- dence of local invasion or metastatic disease. Gadoliniumdiethyl-enetriamine pentaacetic acid-enhanced magnetic resonance imaging has been advocated as a useful diagnostic tool demonstrating heterogenous enhancement of a vascularized tumor. ${ }^{3}$ However, magnetic resonance imaging is not performed during the routine investigative workup for pulmonary thromboembolic disease in most centers. In earlier reports, the diagnosis of primary sarcomas of the PA was often made at postmortem examinations. ${ }^{1}$ With increased surgical intervention for acute and chronic pulmonary obstructive disease in the modern era, the diagnosis is now often made on the basis of histopathologic analysis of the specimens removed during surgical intervention, as in both the described cases. ${ }^{2}$

The prognosis of primary sarcoma of the PA is very poor without treatment. There is a mean survival of only 1.5 months after diagnosis, and death is usually due to pulmonary dysfunction and right heart failure. ${ }^{1}$ Surgical intervention remains the primary treatment. Surgical intervention in the form of pulmonary endarterectomy, resection, and reconstruction of the PA or pneumonectomy have provided significant palliation of symptoms and improved survival. ${ }^{2,4}$ A median survival of 10 months had been reported after such surgical intervention, as well as significant palliation in terms of improvement in quality of life, exercise tolerance, and hemodynamics. ${ }^{1,2}$ Despite aggressive surgical treatment, prolonged tumor-free survival or cure is still rare, with only a few reported cases, while most patients die from recurrent and metastatic diseases. ${ }^{2}$ Treatment of locally advanced PA sarcoma with a combined heart-lung resection followed by heart-lung transplantation has also been attempted. ${ }^{5}$ The utility of such aggressive treatment is limited by the development of metastatic disease and poor survival outcome. ${ }^{5}$ There is little information in the published literature regarding 
the effectiveness of adjuvant therapy for these aggressive tumors. The role of adjuvant radiation therapy is not well defined but is generally advocated because it might decrease the incidence of recurrent tumor. Primary PA sarcoma has a predilection to result in metastatic disease because of its tendency to embolize into the pulmonary arterial tree. Chemotherapy might be effective against microscopic metastatic disease and might decrease the burden of recurrent pulmonary and other systemic disease; however, there has been limited experience with this in the past.

In summary, we present 2 cases of primary PA sarcoma that mimicked pulmonary thromboembolic disease. In both patients the diagnosis of PA sarcoma was made only after pathology was reviewed postoperatively. Despite diagnostic, operative, and oncologic advances over the last few decades, PA sarcoma still remains a diagnostic dilemma associated with a dismal prognosis.

\section{References}

1. Kruger I, Borowski A, Horst M, de Vivie ER, Theissen P, GrossFengels W. Symptoms, diagnosis and therapy of primary sarcoma of the pulmonary artery. Thorac Cardiovasc Surg. 1990;38:91-5.

2. Anderson MB, Kriett JM, Kapelanski DP, Tarazi R, Jamieson SW. Primary pulmonary artery sarcoma: a report of six cases. Ann Thorac Surg. 1995;59:1487-90.

3. Rafal RB, Nichols JN, Markisz JA. Pulmonary artery sarcoma: diagnosis and postoperative follow-up with gadolinium-diethyl-enetriamine pentaacetic acid-enhanced magnetic resonance imaging. Mayo Clin Proc. 1995;70:173-6.

4. Mayer E, Kriegsmann J, Gaumann A, Kauczor HU, Dahm M, Hake U, et al. Surgical treatment of pulmonary artery sarcoma. J Thorac Cardiovasc Surg. 2001;121:77-82.

5. Talbot SM, Taub RN, Keohan ML, Edwards N, Galantowicz ME, Schulman SC. Combined heart and lung transplantation for unresectable primary cardiac sarcoma. J Thorac Cardiovasc Surg. 2002; 124:1145-8.

\section{Surgical repair of a leaking double postinfarction left ventricular pseudoaneurysm}

Predrag Milojevic, MD, FETCS, Vojislava Neskovic, MD, DEAA, Milan Vukovic, MD, Dusko Nezic, MD, PhD, FETCS, and Bosko Djukanovic, MD, PhD, Belgrade, Serbia and Montenegro

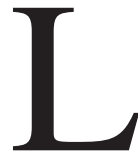

eft ventricular pseudoaneurysm is an uncommon complication of transmural myocardial infarction. It usually forms several weeks after the infarction on the inferior or posterolateral left ventricular wall when cardiac rupture is contained by the adherent pericardium.

Pseudoaneurysm is characterized by a narrow neck in comparison with the diameter of the pseudoaneurysmal sac. Through this sharp discontinuity of myocardial tissue, the pseudoaneurysm communicates with the left ventricular chamber, and bidirectional blood flow can be registered by means of color Doppler echocardiography during the cardiac circle. Considering the risk of secondary rupture or leaking, different treatments are recommended.

We present a case of double inferoposterior left ventricular pseudoaneurysm. To our knowledge, this is the first reported case of double pseudoaneurysm successfully surgically repaired.

\footnotetext{
From the Department of Cardiac Surgery, Dedinje Cardiovascular Institute, Belgrade, Serbia and Montenegro.

Received for publication March 8, 2004; revisions requested March 29, 2004; accepted for publication April 6, 2004.

Address for reprints: Predrag Milojevic, MD, FETCS, Department of Cardiac Surgery II, Dedinje Cardiovascular Institute, M. Tepica 1, 11040 Belgrade, Serbia and Montenegro (E-mail: pmilojevic@ sezampro.yu).

J Thorac Cardiovasc Surg 2004;128:765-7

$0022-5223 / \$ 30.00$

Copyright (C) 2004 by The American Association for Thoracic Surgery

doi:10.1016/j.jtcvs.2004.04.021
}

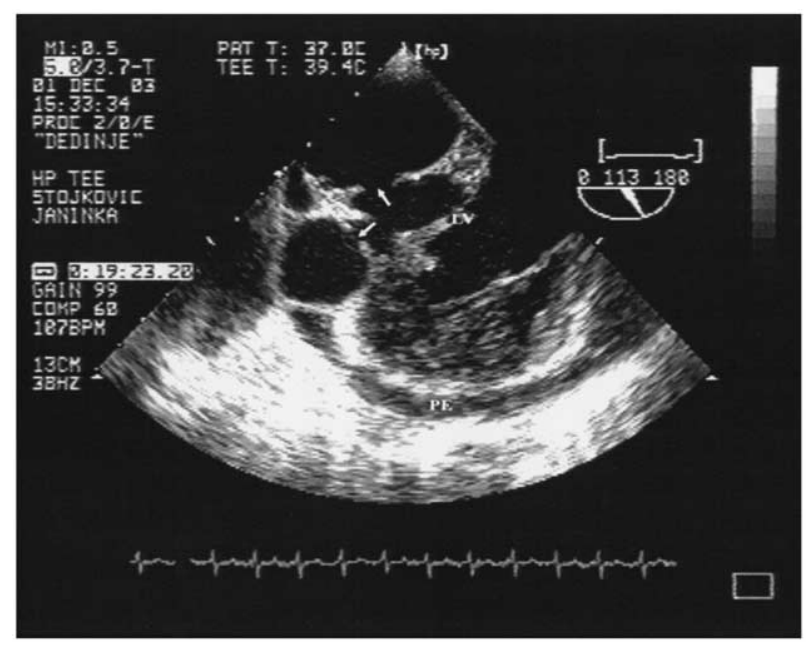

Figure 1. Transesophageal echocardiography: modified longitudinal 2-chamber view. Discontinuity of the inferoposterior myocardial wall at 2 separate places (arrows), with 2 narrow-neck aneurysmal sacs, indicating pseudoaneurysm formation is shown. $L V$, Left ventricle; $P E$, pericardial effusion.

\section{Clinical Summary}

A 45-year-old obese woman was transferred to our institution on an emergency basis with signs of imminent pericardial tamponade. Three months previously, she had acute transmural inferoposterior myocardial infarction that was treated conservatively. At the time 